\title{
CENTER OF FLEXURE OF A TRIANGULAR BEAM
}

\author{
R. N. GOSS
}

1. Introduction. The resolution of Saint-Venant's general problem of a loaded beam into the simpler problems of torsion and pure flexure involves the determination of a load point $P$ in the end section such that the local twist vanishes at a point $Q$ in the section. The position of $P$ in general depends upon the position of $Q$, the choice of which in turn is governed by physical considerations. For example, the local twist may be required to vanish at the load point itself, in which case the point is commonly called the center of shear. For most problems the preferred position of $Q$ is at the centroid, since for this choice the average value of the local twist is zero over the entire section. Moreover, when $Q$ is thus chosen, the point $P$, called the center of flexure of the section, is closely related both to the center of least strain $^{1}$ and to the center of twist ${ }^{2}$ as these terms are usually defined.

The center of flexure has been determined for only a few sections which have uniaxial symmetry. For sections in the form of an isosceles triangle only the cases of the right triangle ${ }^{3}$ and the incompressible equilateral triangle $e^{4}$ have been treated theoretically, while an experimental determination of the center of flexure of a narrow section has been made by Duncan, Ellis, and Scruton in connection with their studies on airfoils. ${ }^{5}$ In this paper the flexure solution of $B . R$. Seth $^{6}$ is used to derive a formula for the center of flexure of any isosceles-triangular section for any value of Poisson's ratio. This formula contains as an unknown factor only the torsional rigidity, for which an approximation has been obtained by Duncan, Ellis, and Scruton by means of the Rayleigh-Ritz method. ${ }^{7}$ With this approximation the formula gives a result in good agreement with the experimental determination of these authors.

Presented to the Society, August 30, 1949; received by the editors June 25, 1949 and, in revised form, October 13, 1949.

1 A. C. Stevenson, Proc. London Math. Soc. (2) vol. 50 (1949) p. 537.

2 A. C. Stevenson, Philos. Trans. Roy. Soc. London. Ser. A vol. 237 (1938) p. 177.

${ }^{3}$ Ibid. pp. 200-205.

${ }^{4}$ S. Timoshenko, Theory of elasticity, 1934, p. 300.

5 W. J. Duncan, D. L. Ellis, and C. Scruton, The Philosophical Magazine (7) vol. 16 (1933) pp. 201-235.

${ }^{6}$ B. R. Seth, Proc. London Math. Soc. (2) vol. 41 (1936) pp. 323-327; Two dimensional potential problems connected with rectilinear boundaries, Lucknow University Studies, no. 13, 1939, pp. 62-65.

${ }^{7}$ Loc. cit. pp. 231-235. 
2. Derivation of the formula. We consider a uniform cantilever beam in the form of a right cylinder of isosceles-triangular cross-section and take the $z$-axis to be that generator which contains the vertices enclosed by the equal sides of the sections (Fig. 1). The

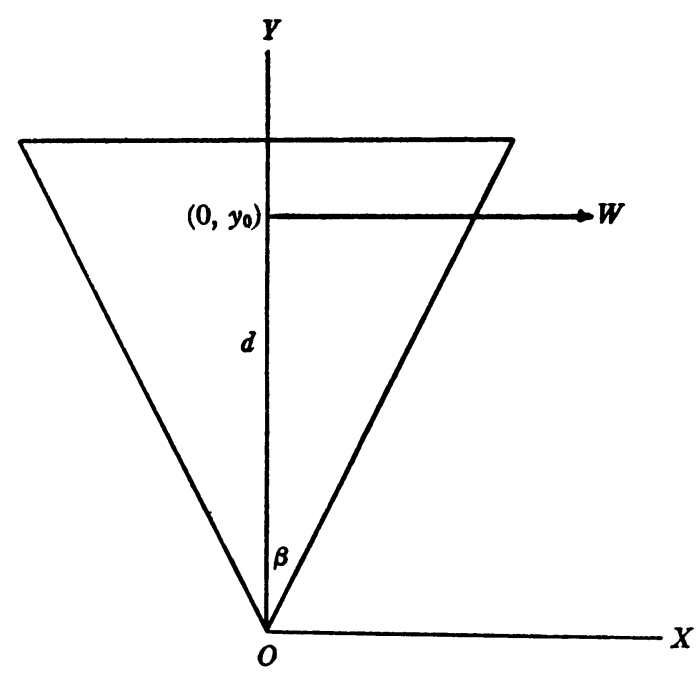

FIG. 1

origin of rectangular coordinates in the plane of the free end is at the apex of the triangular section, and the $y$-axis lies along the axis of symmetry. We denote by $d$ the altitude and by $\beta$ the semi-vertical angle of the triangle. A load of magnitude $W$ is applied parallel to the $x$-axis from the point $\left(0, y_{0}\right)$. For this triangle the nonvanishing stress components which lie in the plane of the.section are given by

$$
\begin{aligned}
& \tau_{z x}=\mu \tau\left(\frac{\partial \phi}{\partial x}-y\right)+\frac{\mu W}{E I}\left[\frac{\partial \chi}{\partial x}-\frac{\sigma x^{2}}{2}-\left(1-\frac{\sigma}{2}\right)\left(y-\frac{2 d}{3}\right)^{2}\right] \\
& \tau_{y z}=\mu \tau\left(\frac{\partial \phi}{\partial y}+x\right)+\frac{\mu W}{E I}\left[\frac{\partial \chi}{\partial y}-x(2+\sigma)\left(y-\frac{2 d}{3}\right)\right]
\end{aligned}
$$

Here $I$ is the moment of inertia with respect to the $y$-axis, $E$ is Young's modulus, $\sigma$ is Poisson's ratio, $\mu$ is the modulus of rigidity, $\tau$ is a constant, $\phi$ is the torsion function, and $\chi$ the flexure function of the section. The lateral surface of the cylinder is supposed free from surface forces, this fact being expressed by the equation

$$
\tau_{z x} \cos (x, n)+\tau_{y z} \cos (y, n)=0,
$$

where $n$ is the direction normal to the surface. 
Seth has shown that the flexure function is given by

$$
\begin{aligned}
x= & \left(A-\frac{4 d}{3}\right) x y+\left[B-\frac{1}{3}\left(1-\frac{\sigma}{2}\right)\right]\left(x^{3}-3 x y^{2}\right) \\
& +\left(H+\frac{2 \sigma d}{3}\right) \phi+\frac{4 d^{2}}{9}\left(1-\frac{\sigma}{2}\right) x
\end{aligned}
$$

where

$$
\begin{aligned}
& A=\frac{d\left[(1+\sigma) \tan ^{2} \beta-\sigma\right]\left(\tan ^{2} \beta+1\right)}{3 \tan ^{2} \beta-1}, \\
& B=\frac{(1-2 \sigma) \tan ^{2} \beta}{3\left(3 \tan ^{2} \beta-1\right)} \\
& B=\frac{d\left[(1+\sigma) \tan ^{2} \beta-\sigma\right]\left(\tan ^{2} \beta-1\right)}{3 \tan ^{2} \beta-1} .
\end{aligned}
$$

According to Stevenson's definition, the center of flexure is that point of loading for which the local twist,

$$
\frac{1}{2 \mu}\left(\frac{\partial \tau_{y z}}{\partial x}-\frac{\partial \tau_{z x}}{\partial y}\right)
$$

vanishes at the centroid of the section. Under this definition $\left(0, y_{0}\right)$ is the center of flexure provided $\tau=0$. Equating the moment about the $z$-axis of the load $W$ applied at $\left(0, y_{0}\right)$ to the torsional couple due to the stresses $\tau_{y z}$ and $\tau_{z x}$, we have

$$
-W y_{0}=\int_{0}^{d} \int_{-y \tan \beta}^{y \tan \beta}\left(x \tau_{y z}-y \tau_{z x}\right) d x d y .
$$

Substituting in (5) the values of $\tau_{y z}$ and $\tau_{z x}$ given by (1) and (2) with $\tau=0$, using (4), and introducing the torsional rigidity $D$, defined by the equation

$$
D=\mu \iint_{B}\left(x \frac{\partial \phi}{\partial y}-y \frac{\partial \phi}{\partial x}+x^{2}+y^{2}\right) d x d y,
$$

we obtain after integration,

$$
\begin{aligned}
y_{0}= & \frac{2 d\left\{5\left[(1+\sigma) \tan ^{2} \beta-\sigma\right]-2(1-2 \sigma)\right\}}{5(1+\sigma)\left(3 \tan ^{2} \beta-1\right)} \\
& -\frac{3(1+\sigma) \tan ^{4} \beta-3 \tan ^{2} \beta+\sigma}{(1+\sigma) \tan ^{2} \beta\left(3 \tan ^{2} \beta-1\right)} \frac{D}{\mu d^{2}} .
\end{aligned}
$$


3. Principal results. It is clear from (7) that the torsional rigidity $D$ lmust be known before $y_{0}$ can be determined. The exact values of $D$ are known only for the isosceles right triangle and the equilateral triangle. ${ }^{8}$ In the former case, using $D=0.10436 \mu d^{4}$, we find for $\beta=\pi / 4$,

$$
\frac{y_{0}}{d}=.6000-.00872 \frac{\sigma}{1+\sigma},
$$

which agrees with Stevenson's result for this section. For the equilateral triangle, $D=\mu d^{4} / 15 \cdot 3^{1 / 2}$. Substitution of this value in (7) with $\beta=\pi / 6$ and $\sigma=1 / 2$ yields $y_{0}=2 d / 3$, showing that in this case the center of flexure coincides with the centroid.

For arbitrary $\sigma$ we find for the equilateral triangle the limiting value

$$
y_{0}=\frac{11+8 \sigma}{15(1+\sigma)} d .
$$

This is a new result, which shows that despite the triaxial symmetry of the figure the center of flexure does not always coincide with the center of gravity. From (1), (2), and (4), using the torsion function for the equilateral triangle ${ }^{9}$ adapted for the boundary shown in Fig. 1, the exact stresses are found to be

$$
\tau_{z x}=\frac{\mu W}{6 E I}\left[(1-2 \sigma)\left(x^{2}-y^{2}\right)-6 x^{2}+2 d y\right], \quad \tau_{y z}=\frac{\mu W}{3 E I}(1+4 \sigma)(d-y) x
$$

These satisfy the boundary condition (3) only on the side $y=d$. On the sides $y= \pm 3^{1 / 2} x$ the stresses satisfy the condition

$$
\int_{C}\left[\tau_{z x} \cos (x, n)+\tau_{y z} \cos (y, n)\right] d s=0,
$$

where the integral is taken along the side. Unless the standard flexure problem is thus relaxed to the extent of permitting a resultant instead of an exact boundary condition to be satisfied on two of the sides, (8) has no significance. Under the generalized condition, however, the triangle still possesses a point which may be called the center of flexure. Its position is shifted from the centroid toward the side on which the exact condition is satisfied by an amount which depends on Poisson's ratio. If the triangle fails to be perfectly equilateral, as

${ }^{8}$ S. Timshenko, loc. cit. pp. 237, 251.

'I. S. Sokolnikoff, Mathematical theory of elasticity, 1946, p. 140. 
is nearly always the case in practice, the exact conditions may be imposed, and the center of flexure will lie very near the centroid for all values of $\sigma$.

Equation (6) shows that $D$ requires for its determination a knowledge of the torsion function $\phi$. Although this function has been obtained by Seth for certain triangular sections, ${ }^{10}$ it is convenient for values of $\beta$ other than $\pi / 4$ to use the approximate formula of Duncan, Ellis, and Scruton,

$$
D=\frac{2 \mu d^{4} \tan ^{3} \beta}{3\left(1-\tan ^{2} \beta\right)} \frac{\left(6 \tan ^{2} \beta+10\right)^{1 / 2}-4 \tan \beta}{\left(6 \tan ^{2} \beta+10\right)^{1 / 2}+4 \tan \beta} .
$$

With this value of $D, y_{0} / d$ has been calculated for various values of $\beta$ as follows:

\begin{tabular}{c|c|c|c}
\hline \hline$\beta$ & $y_{0} / d$ & $\beta$ & $y_{0} / d$ \\
\hline$\frac{\pi}{24}$ & $.7812+.1163 \frac{\sigma}{1+\sigma}$ & $\frac{7 \pi}{24}$ & $.5726-.0075 \frac{\sigma}{1+\sigma}$ \\
$\frac{\pi}{12}$ & $.7401+.0493 \frac{\sigma}{1+\sigma}$ & $\frac{\pi}{3}$ & $.5495-.0055 \frac{\sigma}{1+\sigma}$ \\
$\frac{\pi}{8}$ & $.7060+.0190 \frac{\sigma}{1+\sigma}$ & $\frac{3 \pi}{8}$ & $.5306-.0038 \frac{\sigma}{1+\sigma}$ \\
$\frac{5 \pi}{24}$ & $.6436-.0530 \frac{\sigma}{1+\sigma}$ & $\frac{5 \pi}{12}$ & $.5173-.0018 \frac{\sigma}{1+\sigma}$ \\
$\frac{\pi}{4}$ & $.6000-.0083 \frac{\sigma}{1+\sigma}$ & $\frac{11 \pi}{24}$ & $.5090-.0005 \frac{\sigma}{1+\sigma}$ \\
\hline
\end{tabular}

Fig. 2 displays in graphical form the dependence of the position of the center of flexure on $\sigma$ for selected values of $\beta$. When the vertical angle is small, the position of the center of flexure is displaced from the centroid toward the base with increasing $\sigma$, while the displacement is in the opposite direction when $\beta$ is greater than $\pi / 6$. The shift is most pronounced for small angles. For angles greater than $\pi / 4$ the dependence of the center of flexure on $\sigma$ is negligible, varying from $0.5 \%$ for $\beta=\pi / 4$ to $0.04 \%$ for $\beta=11 \pi / 24$. The practice of

${ }^{10}$ B. R. Seth, Quart. J. Math. Oxford Ser. vol. 5 (1934) pp. 170-171. 
choosing a convenient value of $\sigma$ arbitrarily in the solution of particular problems is thus theoretically justified at least for certain cases.

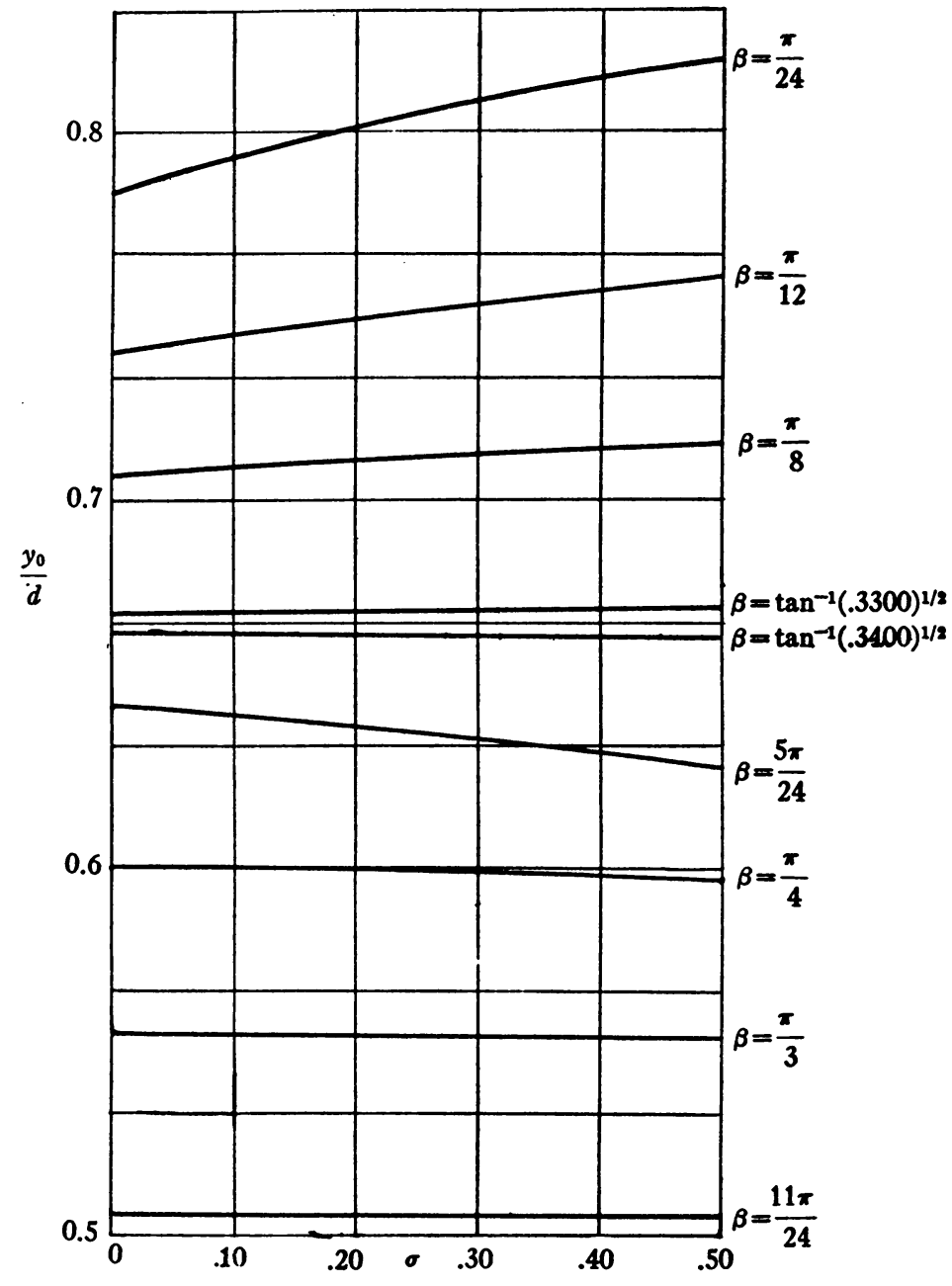

Fig. 2

As a final verification of the validity of (7), we may compare its prediction with the experimental results of Duncan, Ellis, and Scruton on the center of flexure for a steel prism whose cross-section is an isosceles triangle of semi-vertical angle nearly 0.041 radian in magnitude. The measurements, obtained for the single value $\sigma=0.27$, 
were made on sections at various distances from the fixed end of the beam. As extreme values they found $y_{0}=0.857 d$ and $y_{0}=0.814 d$. Formula (7) with $\beta=0.041$ and $\sigma=0.27$ gives $y_{0}=0.8414 d$, a value which agrees well with the experimental results.

Iowa State College of Agriculture and Mechanic Arts

\section{ON THE METRIZATION OF UNIFORM SPACE}

\section{W. COHEN AND CASPER GOFFMAN}

We call a topological space $S$ metrizable by a group $G$ if there is a distance function for $S$ with values in $G$ which defines an equivalent metric topology in $S$. It should be noted that for the triangle inequality required of a distance to have meaning, the group $G$ must be ordered, at least partially. In the classical metrization theorem of Chittenden $[1],{ }^{1} G$ is the additive group of real numbers. The form of this theorem due to Weil [2] is that a necessary and sufficient condition for a uniform space to be metrizable by the group of real numbers is that it satisfy uniformly the first denumerability axiom of Hausdorff. Kalisch [3], using Weil's theorem that a uniform space is equivalent to a subset of a direct product of metric spaces with real distances, showed that every uniform space is metrizable by a partially ordered group. A related result due to Zelinsky [4] gives necessary and sufficient conditions that a topological field have a valuation in an ordered group.

Here we give necessary and sufficient conditions that a uniform space be metrizable by an ordered abelian group $G$. We shall assume that the identity in $G$ is not isolated in the order of $G$, a restriction which excludes the topologically uninteresting discrete spaces. We have shown [5] that the topology of such a group $G$ is determined by a limiting ordinal $\xi^{*}=\xi^{*}(G)$ with the property that if $\eta^{*}<\xi^{*}$ and $\xi_{\eta}$ is a single-valued function on $\eta<\eta^{*}$ to $\xi<\xi^{*}$, then

$$
\sup \left[\xi_{\eta} \mid \eta<\eta^{*}\right]<\xi^{*}
$$

and elements $a_{\xi}, \xi<\xi^{*}$, decreasing in $G$, such that inf $\left[a_{\xi} \mid \xi<\xi^{*}\right]=\theta$, the identity in $G$.

Theorem. A uniform space $S$ is metrizable by an ordered abelian 1949.

Presented to the Society, October 29, 1949; received by the editors September 9,

${ }^{1}$ Numbers in brackets refer to the bibliography at the end of the paper. 